\title{
Calculating Uncertainty in Geodynamic Models of Subduction
}

\author{
$\underline{\text { S. Clark }}^{\text {a }}$ \\ ${ }^{a}$ Computational Geoscience Department, Simula Research Laboratory \\ Martin Linges vei 17, Fornebu, Norway \\ Email: stuart@simula.no
}

\begin{abstract}
Subduction trenches are locations around the world at which one piece of the Earth's crust (the subducting plate) is pushed into the mantle underneath another piece (the overriding plate). Slabs form one of the main sources of heterogeneity of the mantle. Though the present day positions of slabs can be imaged by seismic tomography, to understand slab origins, subduction models have been used. Such models often incorporate regional or global plate reconstructions, showing the location of subduction trenches through time, to accurately predict slab locations in the mantle. Until now, trench locations in such models have been assumed to be exact, though the position of trenches in only inferred by the motion of the overrding plate and other data, such as subduction related volcanism.

In this paper, I ask the question: What if there are inaccuracies in the trench location? What implications would such inaccuracies have for the propagation of uncertainty through the model domain? Drawing upon a statistical approach called the Probabilistic Collocation Method (PCM), I approximate the effect of variability in the trench location by two model simulations with different trench locations, using subduction along the Pacific-Australian plate boundary, at approximately the location of Samoa. Interestingly, the trench location is precisely known at the end of the simulation (in the present day), while its position is least certain at the models initial point, $40 \mathrm{Myr}$ ago.

From this simulation, I calculate the variance of the temperature and velocity fields in the model domain as the model progresses. Measuring this variability as a function of depth, I observe that the variance propagates down into the mantle as a function of time. Using least squares fitting, I calculate that the propagation rate is logarithmic in time. Thus, the growth of the uncertainty is slowed through time as the trench location becomes more precisely defined towards the present.
\end{abstract}

Keywords: Subduction, probabilistic collocation method, uncertainty propagation 


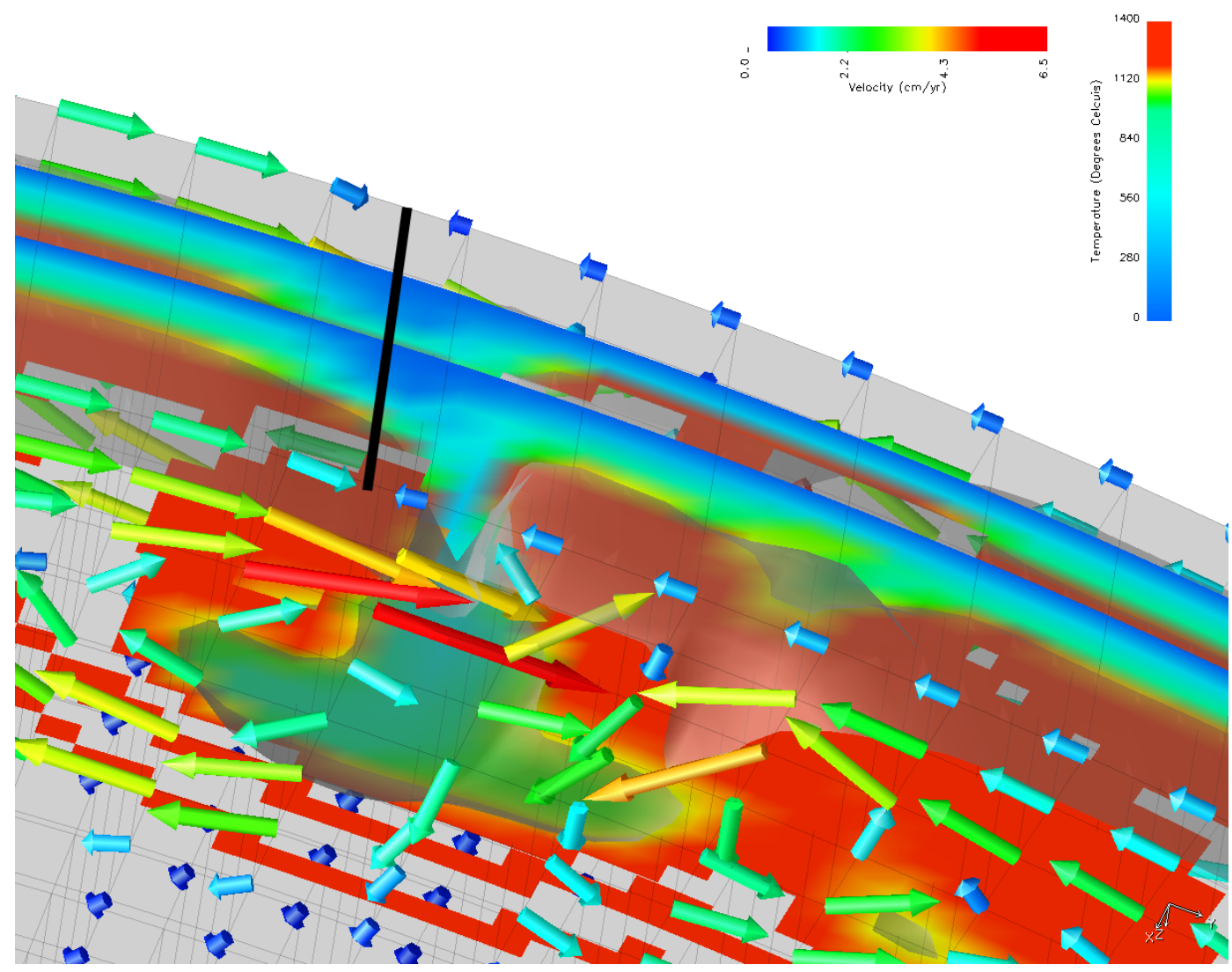

Figure 1: A single realisation of the deterministic model for a given trench position (solid black line) migrating to the left. The model is shown after 20.7 Myrs of model evolution.

\section{INTRODUCTION}

Mid-ocean ridge systems are where new crust is formed as magma cools. As the rocks cool, they store the magnetic polarity of the Earth's magnetic field in a permanent magnetisation. This magnetic signature can be used to calculate the age of the crust, and by removing younger segments, where it formed. Subduction trenches, in which the Earth's crust is pushed into the mantle, destroy the crust and therefore their paleoposition is harder to calculate (Condie, 1997). It may be assumed such trenches are moved with the non-subducting (overriding) plate, but such an assumption may not hold as obduction transfers material from the downgoing plate to the overriding plate (Ribeiro, 2002). In geodynamic models of subduction that include velocities of the plates back through time, having an accurate position for the trench can be crucial for the calculation of the location of slab material deep into the mantle (Clark and Müller, 2008; Stadler et al., 2010). This paper addresses the question of the uncertainty of subduction trench location by allowing the trench position to be a stochastic variable rather than assuming that its location is as accurately known in the past as it is today. The next section addresses both the method of how the mantle convection model is run as well as the assumptions utilised in turning the problem from a deterministic one into a stochastic one. In the third section, I present the results of the calculated variance as well as data relating to its growth and propagation through the model domain. Finally, I draw conclusions about the this growth and propagation based on logarithmic curve fitting. 


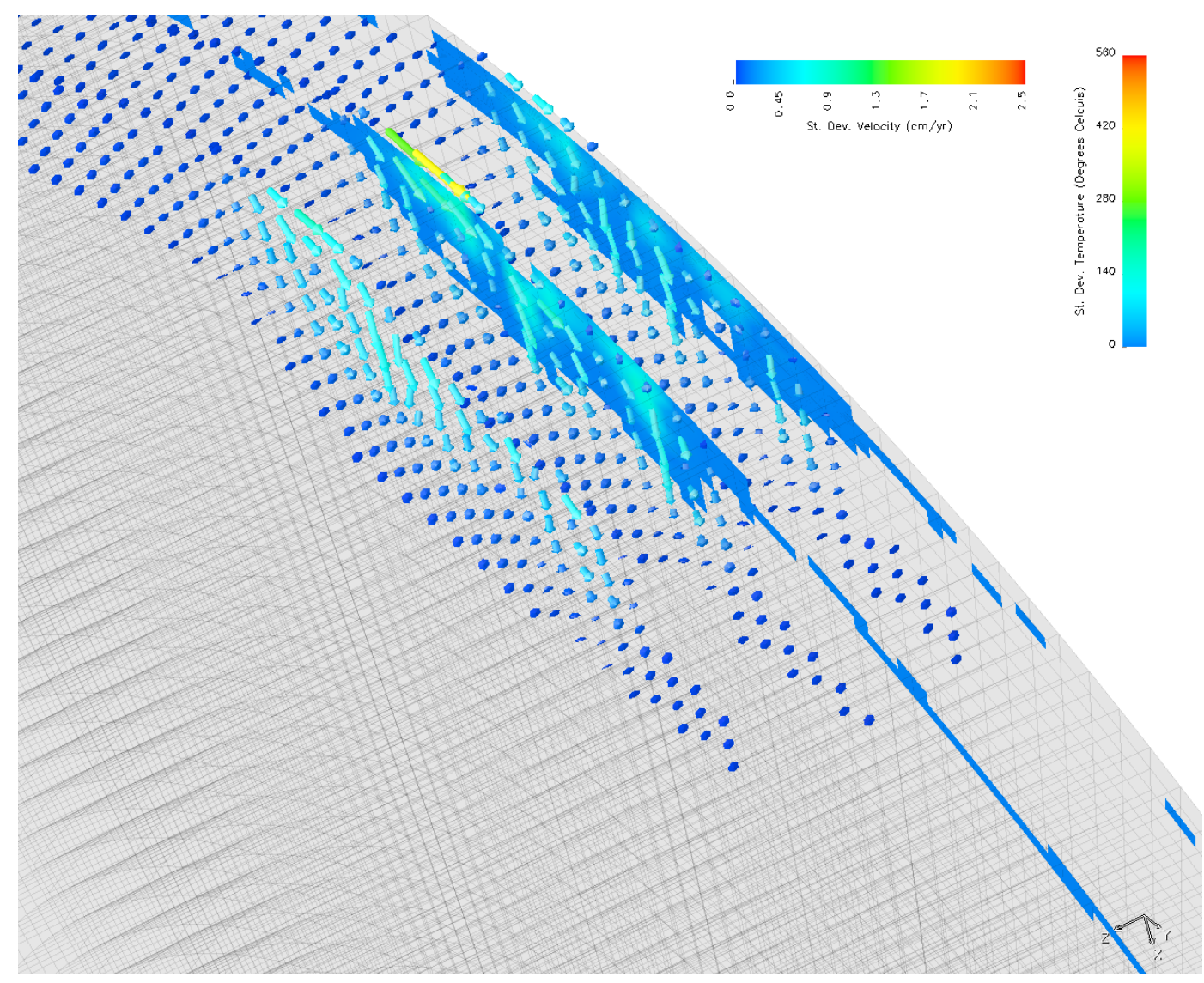

Figure 2: Variation in the temperature (coloured field) and velocity (coloured arrows) at 5 Myrs

\section{Methodology}

\subsection{Mantle Convection}

Following the convention in the geodynamics community to treat the mantle as a fluid over geologic timescale (van Keken et al., 1997; Shubert et al., 2001), I utilise the incompressible Stokes equation coupled with the energy equation neglecting source terms:

$$
\begin{aligned}
-\nabla \cdot \sigma-\nabla p & =R_{a} T e, \\
\nabla \cdot u & =0, \\
\frac{\partial T}{\partial t}+u \cdot T-\kappa \nabla^{2} T & =0 .
\end{aligned}
$$

Where $u$ is the velocity, $p$ is the pressure and the temperature is $T . R_{a}$ is the Rayleigh number, determining the vigour of convection, set at $10^{6}, \kappa$ is the thermal diffusivity, assumed to be $10^{-6} \mathrm{~ms}^{-} 1$ while $e$ is a unit vector in the direction of gravity. $\sigma$ is the deviatoric stress tensor, governed in the Newtonian case by the following:

$$
\sigma=\eta\left(\nabla u+(\nabla u)^{T}\right)
$$

where $\eta$ is the viscosity, for this case $10^{19} \mathrm{~Pa} \mathrm{~s}$. 
The domain is in spherical coordinates, with $161 \times 5 \times 97$ nodes (longitude $\times$ latitude $\times$ depth). The mantle is initially free of thermal anomalies with a constant temperature of $1400^{\circ} \mathrm{C}$ everywhere except near the surface. Close to the surface, the initial terature is given as an exponential decay function from $1400^{\circ} \mathrm{C}$ in the sub-lithospheric mantle to $0^{\circ} \mathrm{C}$ at the surface such that the profile follows that of a $40 \mathrm{Myr}$ old oceanic plate. The surface velocity is imposed, representing two converging tectonic plates, based on the Pacific and Tonga plates, a system for which there has been steady convergence for the last 40 Myrs. As the model evolves, the trench migrates to the west, with convergence rates between $1 \mathrm{~cm} / \mathrm{yr}$ and 16 $\mathrm{cm} / \mathrm{yr}$ (Müller et al., 2008). A solution to the equations after 20.7 Myrs is shown in Figure 1. The trench moves towards the left as the model evolves from 30 Myrs in the past to the present. In the subsequent section, I consider that the trench position has a $2 \%$ uncertainty associated with its position based on the reconstruction from the present. Thus, the uncertainty of the trench position will be largest at the start of the model.

\subsection{Uncertain Plate Reconstructions}

Assume that the position of the trench is uncertain, but governed by a normal probability distribution. Under the assumption of a spherical Earth and of non-deforming plates, paleo-position is typically given as a spherical rotation of the tectonic plate. Such a spherical rotation can be specified by Euler rotations, in which the piece is moved along a great circle defined by a Euler pole $(\alpha, \beta)$. The amount of movement is given by the Euler angle, $\gamma$. For the purpose of investigating uncertainty in plate reconstructions, I assume $\gamma$ is a stochastic variable:

$$
\gamma \sim N(\bar{\gamma}(t), c \bar{\gamma}(t))
$$

Here $\bar{\gamma}(t)$ is the deterministic rotation through time drawn from the relative motion of the two plates and $c$ is a factor the amount of uncertainty in the rotation. For this paper, I set $c=0.02$, a relatively small error, but the further back in time, the larger the uncertainty in the relative displacement of the two plates. Considering a change of variable to a standard normal distribution, $\phi=\frac{\gamma-c \bar{\gamma}}{\bar{\gamma}}$, in the next section I consider an expansion based on $\phi$.

\subsection{Uncertain Mantle Convection}

So that the statistics of the unknowns can be determined as a function of the uncertain trench position, I utilise the idea of orthogonal polynomial expansions from Stochastic Finite Element theory (Ghanem and Spanos, 1991). An order 1 Polynomial Chaos Expansion of a dependent variable $y$ based on an independent variable, $\phi$ is:

$$
y=c_{0} \Gamma_{0}(\phi)+c_{1} \Gamma_{1}(\phi),
$$

where $\Gamma_{\{0,1\}}$ are orthogonal polynomials. Utilising the Probabilistic Collocation Method (Tatang et al., 1997; Loeven and Bijl, 2008), I can then calculate the statistical modes of the unknowns. The Probabilistic Collocation Method specifies that in cases for which $\phi$ is normally distributed, the orthogonal polynomials to be used are the recursively defined Hermite polynomials, for which $\Gamma_{0}=1$ and $\Gamma_{1}(\phi)=\phi$. Thus, the dependent variables of the temperature, $T$ and each component of velocity, $u_{i}$, can be set as a linear expansion of the uncertain variable $\phi$, as follows:

$$
\begin{array}{r}
T(t)=c_{0}^{T}(t)+c_{1}^{T}(t) \phi(t), \\
u_{i}=c_{0}^{u_{i}}(t)+c_{1}^{u_{i}}(t) \phi(t) .
\end{array}
$$

Since these expansions are linear, they require only two realisations of the mantle convection solver at each different time of interest. Since the solution produces both temperature and velocity solutions, I run the complete model for two different values of $\phi$ allowing the coefficients $c_{\{0,1\}}^{\left\{T, u_{i}\right\}}$ to be solved. The Probabilistic Collocation Method specifies that the values of $\phi$ be choosen from the solution to the next 


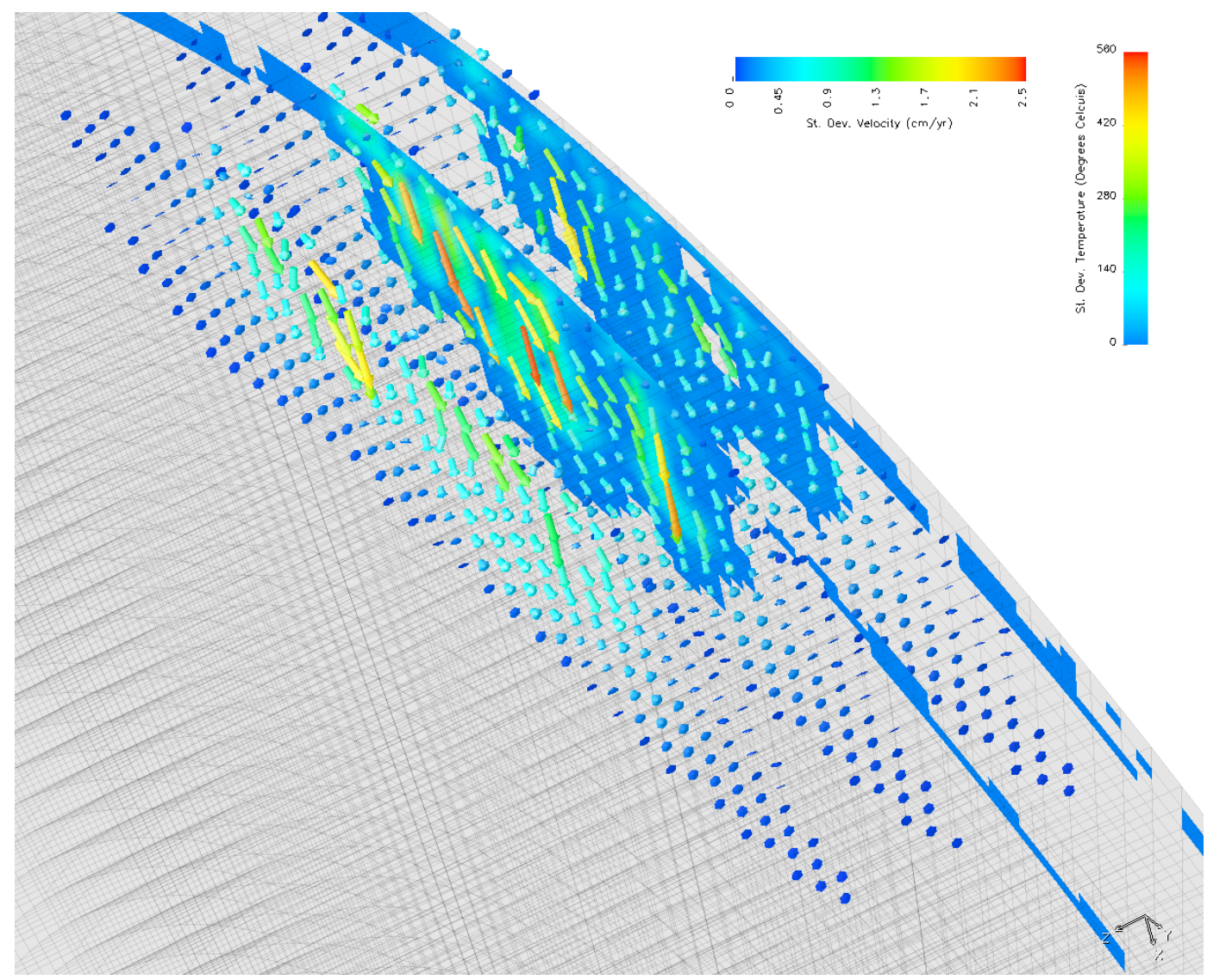

Figure 3: As previous figure, but at 10 Myrs

highest order Hermite polynomial, $\Gamma_{2}$, that is $\phi= \pm 1$. Because $\phi$ is a standard normal distribution, $\mathrm{E}(\phi)$ is zero so the expectation of the temperature and velocity is just $c_{0}^{\left\{T, u_{i}\right\}}$. The variance can then be calculated and, since $\operatorname{Var}(\phi)$ is 1 , the variance of the temperature and velocity is just $\left(c_{1}^{\left\{T, u_{i}\right\}}\right)^{2}$.

\subsection{Results}

Figures 2-4 show three snapshots of the variance in the temperature scalar field and velocity vector field at 5, 10 and 20.7 Myrs respectively. At 5 Myrs, the temperature change is confined to the upper layers and there is only a small variation in velocity. At $10 \mathrm{Myrs}$, there are large velocity variations close to the mean trench position and temperature variations have spread down the domain. At 20.7 Myrs, there is an increased lateral spread of the temperature variations and small velocity variations persist deep into the mantle.

Calculating the maximal depth for which the temperature variations exceeds a very small amount $\left(10^{-5}\right.$ corresponding to about $0.01^{\circ} \mathrm{C}$ ), the results are plotted in Figure 5 for the three snapshots in Figures 2-4. In the same figure (Figure 5), the maximal variances in dimensionless temperature are also plotted (1.0 corresponds to $1400^{\circ} \mathrm{C}$ ). Finally, using least squares logarithmic curve fitting, the best fit curves for each set of data are plotted.

\subsection{Conclusion}

Figure 5 shows that the effect of an order linear uncertainty back through time in the reconstruction angle leads to an order logarithmic propagation of the uncertainty in the temperature field forward in time. This occurs because as the variability in the trench position decreases towards the present, the additional 


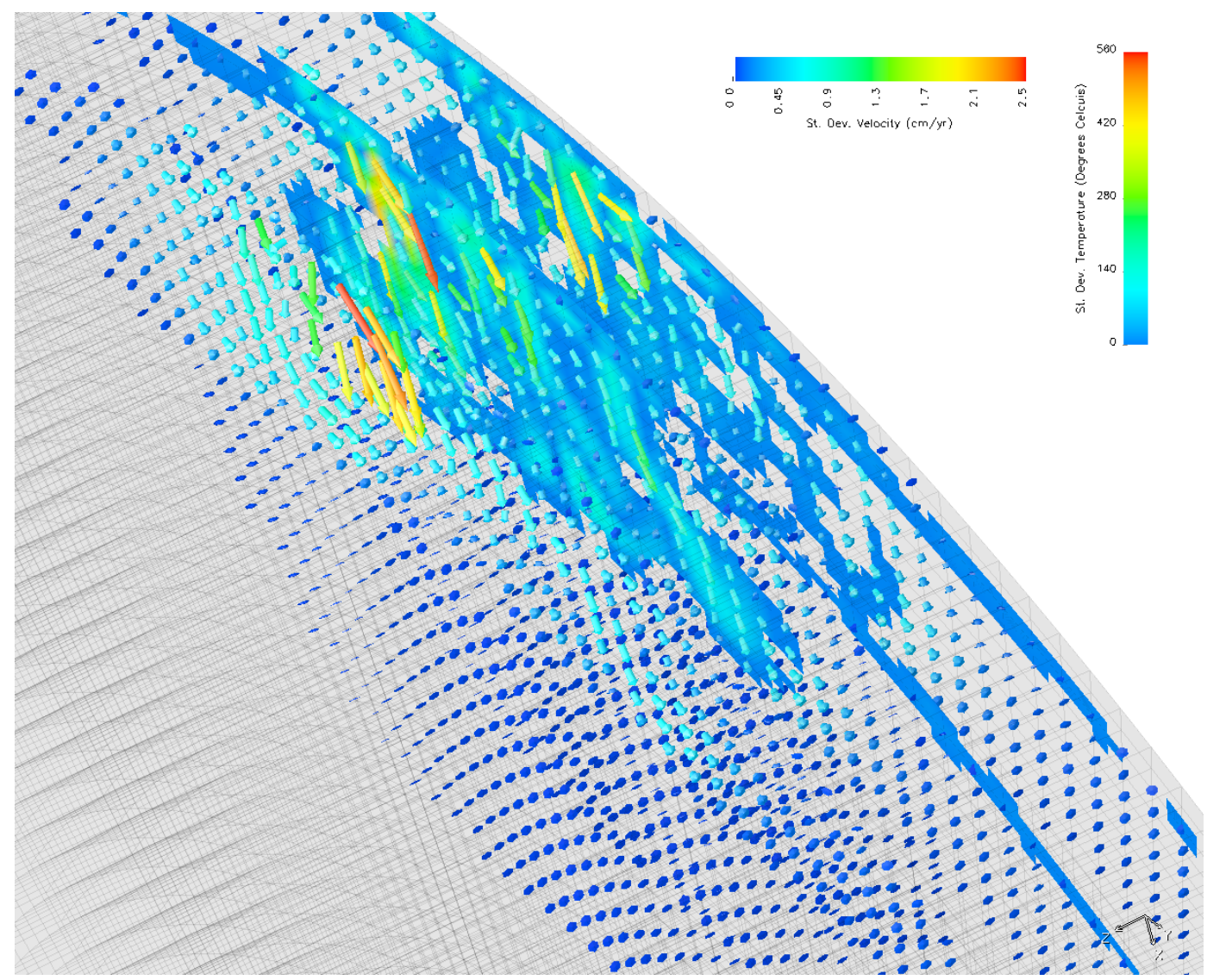

Figure 4: As previous figure, but at 20.7 Myrs

incremental effect of the uncertainty becomes smaller. This implies that both the total uncertainty and the distance the uncertainty get larger in smaller and smaller increments as the model evolves. The ideas in this paper can be further explored by measuring the relationship between the degree of uncertainty and the scaling of the logarithmic dependence as well as the effect of other variables on the uncertainty, such as the mantle viscosity.

\section{ACKNOWLEDgments}

This paper was funded by a Statoil research grant.

\section{REFERENCES}

Clark, S. R. and R. D. Müller (2008). Convection models in the kamchatka region using imposed plate motion and thermal histories. Journal of Geodynamics 46.

Condie, K. C. (1997). Plate Tectonics and Crustal Evolution (4th ed.). Butterworth-Heinemann.

Ghanem, R. G. and P. D. Spanos (1991). Stochastic Finite Elements - a Spectral Approach. Springer.

Loeven, G. and H. Bijl (2008). Airfoil analysis with uncertain geometry using the probabilistic collocation method. In Proceedings of the 49th AIAA Conference on Structures, Structural Dynamics, and Materials Conference, pp. 1-11.

Müller, R. D., M. Sdrolias, C. Gaina, and W. R. Roest (2008). Age, spreading rates and spreading asymmetry of the world's ocean crust. Geochemistry, Geophysics, Geosystems 9. 


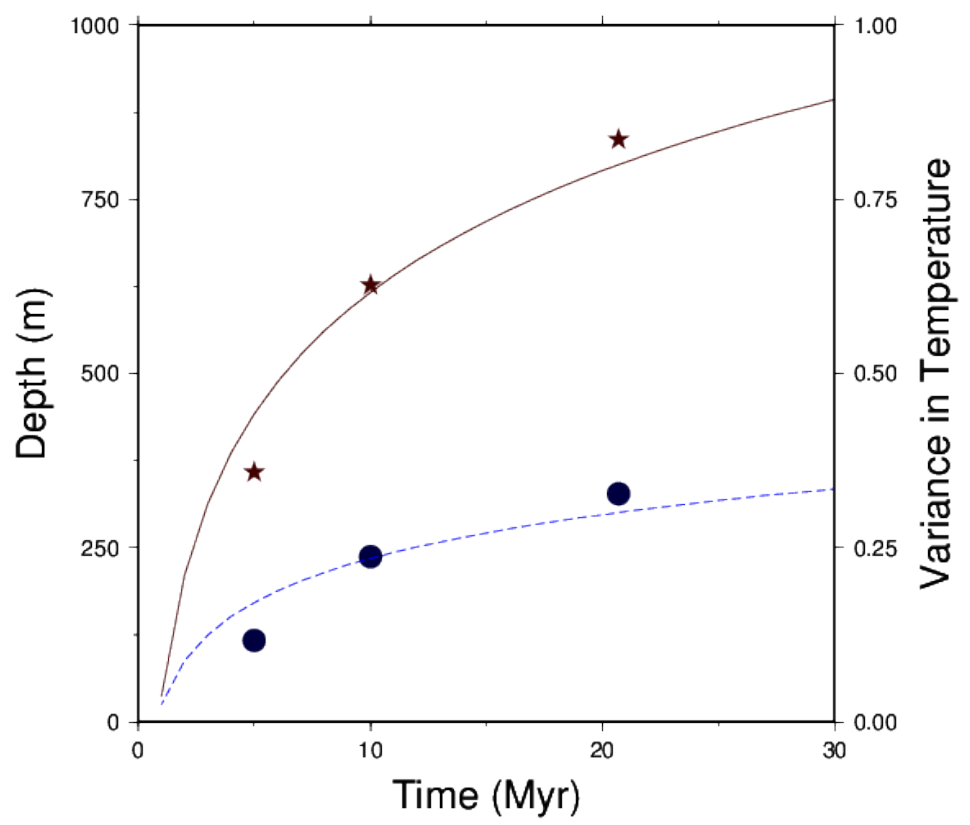

Figure 5: Stars indicate the maximum depth $(d)$ of uncertainty into the model for which the variance of the temperature exceeds $10^{-5}$, while circles are the maximum variance of temperature in the model. Lines are least-squares fitted logarithmic function of the form $d=a+b \ln t$. Solid line is a fit for the maximum depth, for which $a$ is 36.7 and $b$ is 251.8 , while the dashed line is a fit for the maximum variance for which $a$ is 0.025 and $b$ is 0.091 .

Ribeiro, A. (2002). Soft Plate and Impact Tectonics. Springer.

Shubert, G., D. Turcotte, and P. Oloson (2001). Mantle convection in the Earth and planets, pp. 956. Cambridge University Press. 956pp.

Stadler, G., M. Gurnis, C. Burstedde, L. C. Wilcox, L. Alisic, and O. Ghattas (2010). The dynamics of plate tectonics and mantle flow: From local to global scales. Science 329, 1033-1038.

Tatang, M. A., W. Pan, R. G. Prinn, and G. J. McRae (1997). An efficient method for parametric uncertainty analysis of numerical geophysical models. Journal of Geophysical Research 102.

van Keken, P., S. King, H. Schmeling, et al. (1997). A comparison of methods for the modeling of thermochemical convection. Journal of Geophysical Research 102(B10), 22477-22495. 